\title{
State-Of-Charge Estimation for Lithium-Ion Battery Using Improved DUKF Based on State-Parameter Separation
}

\author{
Chuan-Xiang Yu*, Yan-Min Xie, Zhao-Yu Sang, Shi-Ya Yang and Rui Huang \\ State Key Laboratory of Power Transmission Equipment \& System Security and New Technology, Chong Qing \\ University, Chongqing 400030, China; 201711131140@cqu.edu.cn (Y.-M.X.); 201811131077@cqu.edu.cn (Z.-Y.S.); \\ 201811131102@cqu.edu.cn (S.-Y.Y.); 201911131138@cqu.edu.cn (R.H.) \\ * Correspondence: ychx@cqu.edu.cn
}

Received: 12 September 2019; Accepted: 1 October 2019; Published: 23 October 2019

check for updates

\begin{abstract}
State-of-charge estimation and on-line model modification of lithium-ion batteries are more urgently required because of the great impact of the model accuracy on the algorithm performance. This study aims to propose an improved DUKF based on the state-parameter separation. Its characteristics include: (1) State-Of-Charge (SoC) is treated as the only state variable to eliminate the strong correlation between state and parameters. (2) Two filters are ranked to run the parameter modification only when the state estimation has converged. First, the double polarization (DP) model of battery is established, and the parameters of the model are identified at both the pulse discharge and long discharge recovery under Hybrid Pulse Power Characterization (HPPC) test. Second, the implementation of the proposed algorithm is described. Third, combined with the identification results, the study elaborates that it is unreliable to use the predicted voltage error of closed-loop algorithm as the criterion to measure the accuracy of the model, while the output voltage obtained by the open-loop model with dynamic parameters can reflect the real situation. Finally, comparative experiments are designed under HPPC and DST conditions. Results show that the proposed state-parameter separated IAUKF-UKF has higher SoC estimation accuracy and better stability than traditional DUKF.
\end{abstract}

Keywords: lithium-ion batteries; SoC estimation; state-parameter separation; improved dual unscented Kalman filter

\section{Introduction}

In recent years, the automobile industry has gradually developed, and the contradiction between traditional fuel vehicles and environmental carrying capacity has intensified. The demand of replacing traditional fuel vehicles with new energy ones is becoming more and more urgent. Lithium-ion batteries are widely used in pure electric vehicles as core power sources due to their high energy density and long cycle life. State-Of-Charge $(\mathrm{SoC})$ is defined as the percentage of the current residual capacity of batteries. Accurate estimation of SoC can not only be used as an important basis for drivers to judge the endurance of batteries, but also for achieving equalization control, overcharge and discharge protection, overheat protection and other functions in battery management system. However, as an artificially defined variable, SoC can only be calculated by voltage, current and temperature of batteries [1]. Based on this, the methods for SoC estimation in recent years can be divided into those based on characterization parameters and definitions, data-driven and battery modeling theory [2].

Methods based on characterization parameters and definitions include impedance method $[3,4]$, open circuit voltage method [5,6] and ampere-time counting method [7]. It directly estimates by establishing a simple correspondence between the characterization parameters and the SoC or by 
using the ampere-time definition. The data-driven method such as neural network [8], support vector machine [9] and fuzzy theory [10] utilizes a large amount of offline data to train a mapping relationship between battery current, voltage, temperature and SoC. The method based on battery modeling theory relies on establishing a high-accuracy model for the battery, and then using the filtering theory to estimate the state variables in closed-loop. Among these, the method based on battery modeling theory has larger application prospects due to its good accuracy and real-time performance.

Currently, the equivalent circuit model is more suitable for real-time systems because of its simple structure and fewer parameters. The parameter identification methods are mainly divided into off-line method and on-line method. Off-line method usually uses Hybrid Pulse Power Characterization (HPPC) experiment to identify the parameters in different SoC stages, but this method identifies only at pulse charging and discharging which proves that the parameters obtained are not representative even for the same SoC. Online methods are generally based on system automatically identification theory, including recursive least squares (RLS) method and its derivative algorithm [11], Kalman filter (KF) and its derivative algorithm [12-15]. These methods also use the external data of batteries, combined with the state estimation and modify the model parameters at the same time. Dave Andre proposed a dual filter algorithm for simultaneous estimation of internal resistance and SoC of batteries by combining KF and unscented Kalman filter (UKF) [16]. Feng Guo designed a multi-scaled Dual EKF (DEKF) to estimate the SoC and modify all model parameters at the same time [17]. Xu Zhang proposed the method of EKF-UKF based on the data-driven model [18]. In this algorithm, the EKF is used to identify the battery model parameters and the UKF is employed to estimate the battery SoC. Qianqian Wang proposed a Dual UKF (DUKF) to simultaneously estimate the polarization parameters, internal resistance and SoC of batteries [19]. Zewang Chen analyzed the influence of different model parameters on the accuracy of the model, and then designed a dual UKF algorithm to simultaneously estimate the internal resistance and $\mathrm{SoC}$ of batteries [20]. Among the above algorithms, the accuracy of the model is evaluated by comparing the predicted voltage with the observation. This method will bring unreliable judgment due to the self-correcting characteristics of the closed-loop algorithm. These dual filter algorithms are carried out regardless of the sequence, which easily leads to divergence of the results when the state is inaccurate. All the above algorithms take polarization voltage as state variable, however, it has a strong correlation with polarization parameters, which will reduce the accuracy of state estimation.

Considering the above problems, an improved dual-unscented Kalman filter algorithm based on state-parameter separation is proposed in this paper. The algorithm takes SoC as the only state variable and estimates the model parameters at the same time, which improves the accuracy. In this algorithm, two filters are ranked to solve the poor stability caused by inaccurate initial values of state variables in dual filter algorithms. In this study, the open-loop model output is used as the criterion to evaluate the accuracy of the model. The arrangement of the paper is as follows: Section 2 mainly introduces the equivalent circuit model adopted in this paper and the off-line parameter identification method; Section 3 introduces the implementation details of the improved dual unscented Kalman filter algorithm based on state-parameter separation; Section 4 introduces the relevant experiments; Section 5 proves that evaluating the model accuracy by comparing the predicted voltage of the closed-loop algorithm with experimental data is unreliable, then the SoC estimation and parameter modification accuracy of the proposed algorithm are also discussed. Section 6 introduces the conclusions of this study.

\section{Battery Model and Off-Line Parameter Identification Method}

Considering the accuracy and complexity of the model, the double polarization (DP) model based on second-order resistor-capacitor (RC) network is chosen in this study [21]. The structure of the circuit is shown in Figure 1. 


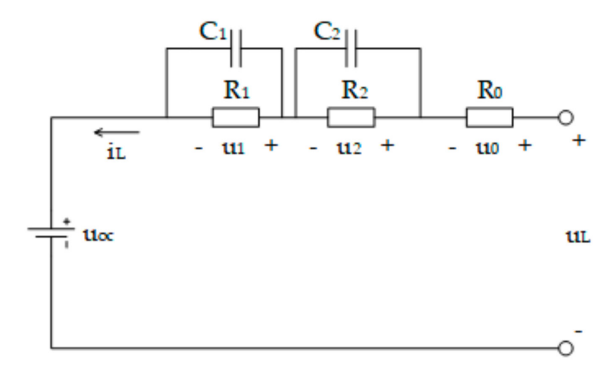

Figure 1. Double polarization (DP) model.

$u_{\mathrm{oc}}$ represents the open-circuit voltage of the battery and its value is a function of SoC. $\mathrm{R}_{0}$ represents the ohmic internal resistance. $R_{1}, R_{2}, C_{1}, C_{2}$ represents the polarization parameters. $i_{L}$ represents the charging current. $\mathrm{u}_{1}, \mathrm{u}_{2}$ represent two polarization voltages. $\mathrm{u}_{0}$ represent the voltage of $\mathrm{R}_{0}$, $\mathrm{u}_{\mathrm{L}}$ represents the terminal voltage of the battery model.

The continuous-time equation of the DP model can be obtained by using Kirchhoff's law and shown as follows.

$$
\begin{gathered}
\left\{\begin{array}{l}
\dot{u_{1}}(t)=\frac{1}{\tau_{1}} \mathrm{u}_{1}(\mathrm{t})+\frac{\mathrm{R}_{1}}{\tau_{1}} \mathrm{i}_{\mathrm{L}}(\mathrm{t}) \\
\dot{\mathrm{u}_{2}}(\mathrm{t})=\frac{1}{\tau_{2}} \mathrm{u}_{2}(\mathrm{t})+\frac{\mathrm{R}_{2}}{\tau_{2}} \mathrm{i}_{\mathrm{L}}(\mathrm{t}) \\
\mathrm{SoC}(\mathrm{t})=\operatorname{SoC}\left(\mathrm{t}_{0}\right)+\int_{t_{0}}^{t} \frac{\mathrm{i}_{\mathrm{L}}(\mathrm{t}) \mathrm{dt}}{\mathrm{Q}_{\mathrm{N}}}
\end{array}\right. \\
\mathrm{u}_{\mathrm{L}}(\mathrm{t})=\mathrm{u}_{1}(\mathrm{t})+\mathrm{u}_{2}(\mathrm{t})+\mathrm{R}_{0} \mathrm{i}_{\mathrm{L}}(\mathrm{t})+\mathrm{u}_{\mathrm{oc}}(\mathrm{SoC})
\end{gathered}
$$

SoC $\left(t_{0}\right)$ is the initial value of $S o C, Q_{N}$ is the nominal capacity of the battery. $\tau_{1}=R_{1} C_{1}, \tau_{2}=R_{2} C_{2}$.

Off-line identification of model parameters is the basis of establishing on-line parameter estimators. Referred to [22], HPPC experiment can identify the parameters of equilibrium potential and over-potential characteristic simultaneously. The specific experimental method is shown in Section 4 . Some experimental scatters of $\mathrm{u}_{\mathrm{oc}}$ are obtained by recording the static ending voltage of different $\mathrm{SoC}$ in HPPC experiments. Then the scatters are fitted by the six-order polynomial shown in Equation (3). $\mathrm{K}_{\mathrm{i}}$ are the constant parameters that need to be identified.

$$
\mathrm{u}_{\mathrm{oc}}(\mathrm{SoC})=\sum_{\mathrm{i}=0}^{6} \mathrm{~K}_{\mathrm{i}} \mathrm{SoC}^{\mathrm{i}}
$$

Ohmic internal resistance and polarization parameters can be identified by the response curve of pulse discharge as shown in Figure 2a. $R_{0}$ can be calculated by the instantaneous change of voltage when current switching on and off as shown in Equation (4). $I_{d}$ is the pulse discharge current. $u_{A}, u_{B}$, $\mathrm{u}_{\mathrm{C}}$ and $\mathrm{u}_{\mathrm{D}}$ are the voltage values at points $\mathrm{A}, \mathrm{B}, \mathrm{C}$ and $\mathrm{D}$ respectively. Because of the short discharge time between $B$ and $C, u_{o c}$ can be considered to be constant, and the terminal voltage can be expressed by Equation (5). The terminal voltage of the discharge recovery interval $\mathrm{D}$ to $\mathrm{E}$ can be expressed by Equation (6), where $\mathrm{u}_{1}(0)$ and $\mathrm{u}_{2}(0)$ represent the polarization voltage at the beginning. Equation (6) is used to fit the experimental data to get $\tau_{1}$ and $\tau_{2}$, then get $R_{1}$ and $R_{2}$ in the same way according to Equation (5).

$$
\begin{gathered}
\mathrm{R}_{0}=\frac{\left(\mathrm{u}_{\mathrm{A}}-\mathrm{u}_{\mathrm{B}}\right)+\left(\mathrm{u}_{\mathrm{D}}-\mathrm{u}_{\mathrm{C}}\right)}{2 \mathrm{I}_{\mathrm{d}}} \\
\mathrm{u}_{\mathrm{L}}=\mathrm{u}_{\mathrm{oc}}+\mathrm{R}_{0} \mathrm{I}_{\mathrm{d}}+\mathrm{R}_{1} \mathrm{I}_{\mathrm{d}}\left(1-\mathrm{e}^{-\mathrm{t} / \tau_{1}}\right)+\mathrm{R}_{2} \mathrm{I}_{\mathrm{d}}\left(1-\mathrm{e}^{-\mathrm{t} / \tau_{2}}\right) \\
\mathrm{u}_{\mathrm{L}}=\mathrm{u}_{\mathrm{oc}}+\mathrm{u}_{1}(0) \mathrm{e}^{-\mathrm{t} / \tau_{1}}+\mathrm{u}_{2}(0) \mathrm{e}^{-\mathrm{t} / \tau_{2}}
\end{gathered}
$$

In order to make full use of the experimental data and take full account of the dynamic changes of model parameters, parameter identification was also carried out in the long discharge recovery in HPPC experiment in this study. Long discharge recovery refers to the voltage recovery of the battery 
after a long discharge which aims to test the pulse characteristics of the next SoC under HPPC test as shown in Figure $2 b . \tau_{1}$ and $\tau_{2}$ can be obtained by fitting the curve from $G$ to $H$. Because $u_{o c}$ cannot be regarded as a constant in the long discharge process, Equation (5) is no longer suitable for curve fitting; $R_{1}$ and $R_{2}$ are not identified here.

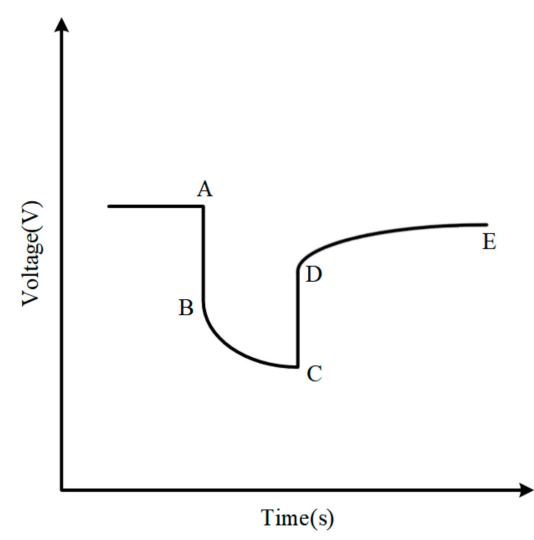

(a)

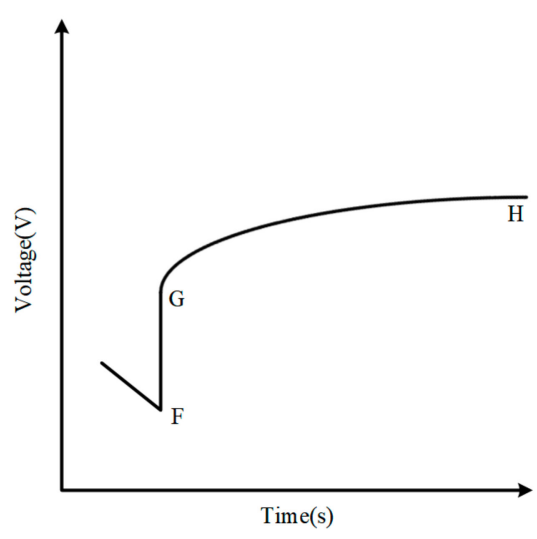

(b)

Figure 2. (a) Voltage response curve under pulse discharge; (b) voltage response curve under long discharge recovery.

\section{Implementation of the State-Parameter Separated IAUKF-UKF}

\subsection{State-Parameter Separated Equation for Dynamic Battery Model}

Since the observation is the battery terminal voltage, SoC is generally estimated together with the polarization voltage in the model [16-20]. However, it can be seen from Equation (1) that there is a strong correlation between polarization voltage and polarization parameters, which results in an enhanced correlation between state and parameters when using the dual filters. The experimental results in Section 5 of this study show that this will reduce the accuracy of state estimation and parameter modification. In this paper, $\mathrm{SoC}$ is considered as the only state variable and parameters as dynamic ones to be modified. The discrete model with noise is organized into Equation (7). It should be declared that that the sampling time is $1 \mathrm{~s}$.

$$
\left\{\begin{array}{l}
\mathrm{SoC}_{\mathrm{k}}=\mathrm{F}\left(\mathrm{SoC}_{\mathrm{k}-1}, \mathrm{i}_{\mathrm{L}, \mathrm{k}}\right)+\omega_{\mathrm{k}}^{\mathrm{SoC}}=\mathrm{SoC}_{\mathrm{k}-1}+\frac{1}{\mathrm{Q}_{\mathrm{N}}} \mathrm{i}_{\mathrm{L}, \mathrm{k}}+\omega_{\mathrm{k}}^{\mathrm{SoC}} \\
\theta_{\mathrm{k}}=\theta_{\mathrm{k}-1}+\omega_{\mathrm{k}}^{\theta} \\
\mathrm{u}_{\mathrm{L}, \mathrm{k}}=\mathrm{G}\left(\mathrm{SoC}_{\mathrm{k}}, \theta_{\mathrm{k}}\right)+v_{\mathrm{k}}=\mathrm{H}_{\mathrm{k}} \cdot \theta_{\mathrm{k}}+\mathrm{u}_{\mathrm{oc}}\left(\mathrm{SoC}_{\mathrm{k}}\right)+v_{\mathrm{k}}
\end{array}\right.
$$

where,

$$
\begin{gathered}
\theta_{\mathrm{k}}=\left[\begin{array}{lllll}
\lambda_{1, \mathrm{k}} & \lambda_{2, \mathrm{k}} & \lambda_{3, \mathrm{k}} & \lambda_{4, \mathrm{k}} & \mathrm{R}_{0, \mathrm{k}}
\end{array}\right]^{\mathrm{T}} \\
\lambda_{1, \mathrm{k}}=\frac{\tau_{1, \mathrm{k}}}{1+\tau_{1, \mathrm{k}}}, \lambda_{2, \mathrm{k}}=\frac{\mathrm{R}_{1, \mathrm{k}}}{1+\tau_{1, \mathrm{k}}}, \lambda_{3, \mathrm{k}}=\frac{\tau_{2, \mathrm{k}}}{1+\tau_{2, \mathrm{k}}}, \lambda_{4, \mathrm{k}}=\frac{\mathrm{R}_{2, \mathrm{k}}}{1+\tau_{2, \mathrm{k}}} \\
\mathrm{H}_{\mathrm{k}}=\left[\begin{array}{lllll}
\mathrm{u}_{1, \mathrm{k}-1} & \mathrm{i}_{\mathrm{L}, \mathrm{k}} & \mathrm{u}_{2, \mathrm{k}-1} & \mathrm{i}_{\mathrm{L}, \mathrm{k}} & \mathrm{i}_{\mathrm{L}, \mathrm{k}}
\end{array}\right]
\end{gathered}
$$

It should be noted that $\mathrm{u}_{1, \mathrm{k}-1}$ and $\mathrm{u}_{2, \mathrm{k}-1}$ in data matrix $\mathrm{H}_{\mathrm{k}}$ are not state variables, but historical data of $u_{1, k}$ and $u_{2, k}$, as shown in Equation (11).

$$
\left\{\begin{array}{l}
\mathrm{u}_{1, \mathrm{k}}=\lambda_{1, \mathrm{k}} \cdot \mathrm{u}_{1, \mathrm{k}-1}+\lambda_{2, \mathrm{k}} \cdot \mathrm{i}_{\mathrm{L}, \mathrm{k}} \\
\mathrm{u}_{2, \mathrm{k}}=\lambda_{3, \mathrm{k}} \cdot \mathrm{u}_{2, \mathrm{k}-1}+\lambda_{4, \mathrm{k}} \cdot \mathrm{i}_{\mathrm{L}, \mathrm{k}}
\end{array}\right.
$$


The structure of this Equation can be further illustrated by Figure 3. At k-moment, model output voltage $u_{\mathrm{L}, \mathrm{k}}$ is determined by state $\mathrm{SoC}_{\mathrm{k}}$ and model parameter $\theta_{\mathrm{k}}$. The former decides $\mathrm{u}_{\mathrm{oc}, \mathrm{k}}$ according to the function $\mathrm{u}_{\mathrm{oc}}(\mathrm{SoC})$, and the latter decides $\mathrm{u}_{1, \mathrm{k}}, \mathrm{u}_{2, \mathrm{k}}, \mathrm{u}_{0, \mathrm{k}}$ together with data matrix $\mathrm{H}_{\mathrm{k}}$. This means that state and model parameters determine different output regions respectively in the process of equation iteration. The advantage of this method is that it can eliminate the strong correlation between state and model parameters, and lay the foundation for using dual filters.

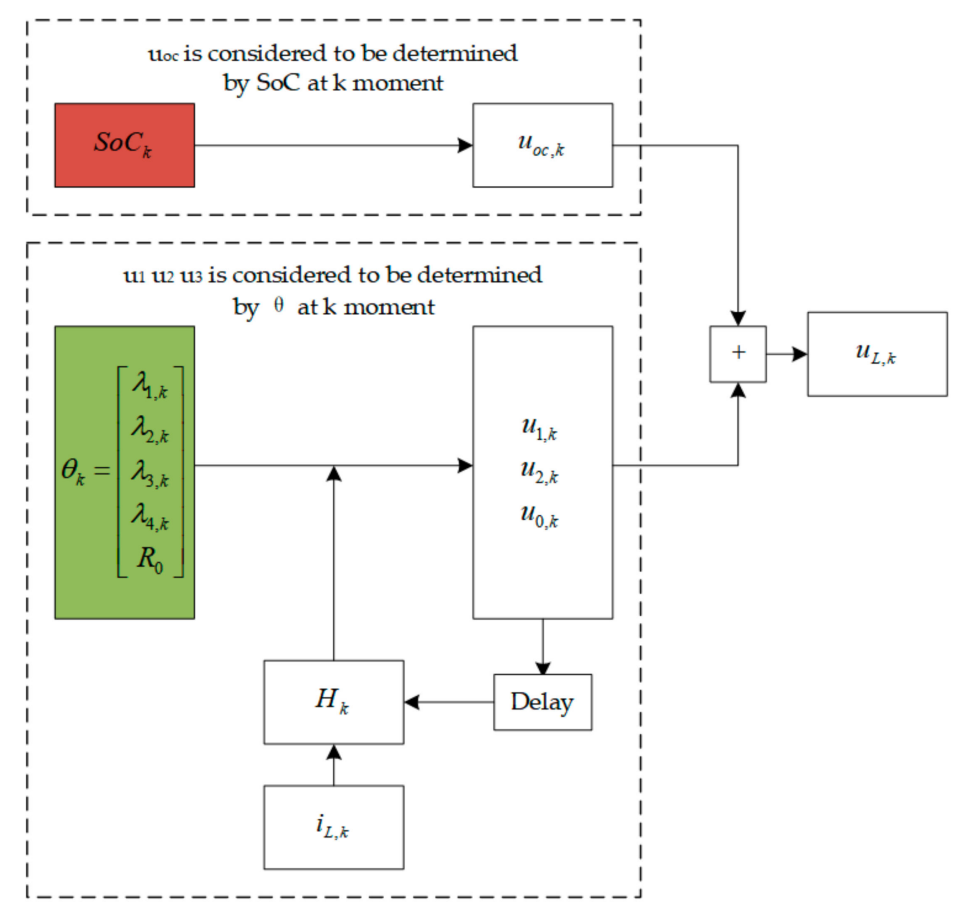

Figure 3. Structure of the state-parameter separated equation.

\subsection{Improved Dual-Unscented Kalman Filter (IDUKF) Algorithm}

Kalman filter algorithm combines the prediction of discrete dynamic system with the observation containing noise, and calculates the model state recursively to get its maximum likelihood estimation [23]. As a non-linear form of traditional KF, UKF improves the accuracy of approximating non-linearity compared with EKF only by first-order Taylor expansion [24]. However, because the estimation accuracy of the whole algorithm still depends on the accuracy of the dynamic model, in order to ensure that the prediction of the model can always track the observation, the algorithm may overcorrect the state and reduce the estimation accuracy in case of inadequate model accuracy, which will be analyzed in Section 5. Therefore, it is necessary to use the theory of system identification combined with external data to modify the model, and run simultaneously with the state estimator. In this study, Adaptive UKF (AUKF) is used as the state estimator by combining Sage-Husa adaptive filtering theory [25] with traditional UKF, and another UKF is used as the modifier of model parameters.

Another advantage of KF is that it can automatically converge the inaccurate initial state to real value. However, it is found that the convergence performance of the traditional dual Kalman filter is weak when the initial state value is inaccurate, which will be analyzed in Section 5. In order to solve this problem, two filters are ranked in this study: when the error $\mathrm{e}_{\mathrm{r}}$ between the filter prediction and the observation is greater than a boundary value $\delta$, the state estimator runs and corrects the inaccurate state, while the parameter modifier stops and holds the parameters still. When the state converges to the real value, the algorithm prediction also converges to the observation. When $\mathrm{e}_{\mathrm{r}}$ is less than or equal to the boundary value, open the parameter modifier and run simultaneously with the state estimator until the next $e_{r}>\delta$ appears. The structure of proposed IAUKF-UKF is illustrated in Figure 4. 


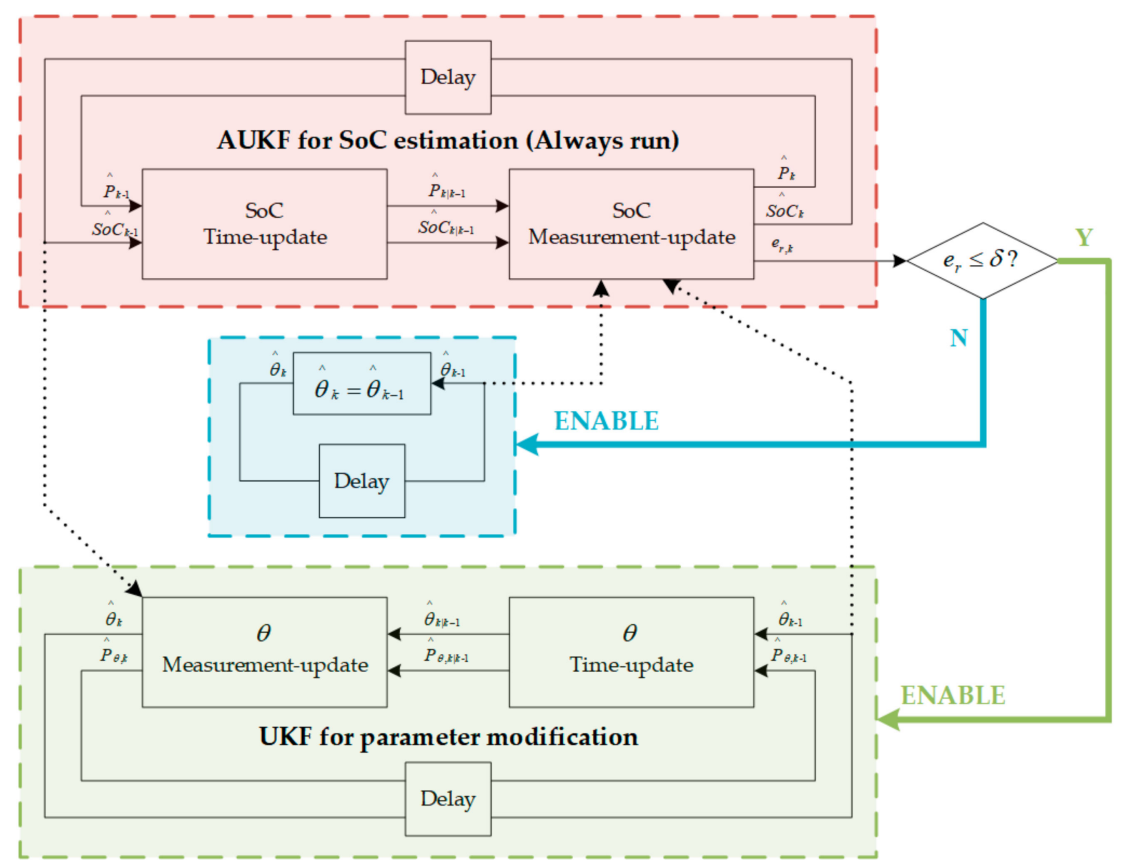

Figure 4. Structure of the state-parameter separated equation structure of IAUKF-UKF algorithm.

Combined with Equations (8) (12), the implementation steps of IAUKF-UKF algorithm based on state-parameter separation are shown as follows.

Step 1: Initializing.

$$
\begin{gathered}
\text { SoC }_{0}=\mathrm{E}\left(\mathrm{SoC}_{0}\right), \mathrm{P}_{\mathrm{SoC}, 0}=\mathrm{E}\left[\left(\mathrm{SoC}_{0}-\hat{\mathrm{SoC}}_{0}\right)\left(\mathrm{SoC}_{0}-\mathrm{SoC}_{0}\right)^{\mathrm{T}}\right] \\
\hat{\theta_{0}}=\mathrm{E}\left(\theta_{0}\right), \hat{\mathrm{P}_{\theta, 0}}=\mathrm{E}\left[\left(\theta_{0}-\hat{\theta_{0}}\right)\left(\theta_{0}-\hat{\theta_{0}}\right)^{\mathrm{T}}\right] \\
\mathrm{QSoC}, 0^{-5}=10^{-5}, \mathrm{Q}_{\theta}=\mathrm{I}_{5} \\
\mathrm{R}_{0}=10^{-3}
\end{gathered}
$$

where $\mathrm{I}_{5}$ means 5-order unit matrix.

Step 2: Time-update for SoC at $\mathrm{k}$-moment, $\mathrm{k}=1,2 \ldots$

First sigma point calculating:

$$
\left\{\begin{aligned}
\sigma_{\mathrm{k}-1}^{\mathrm{i}} & =\mathrm{SoC}_{\mathrm{k}-1}, \mathrm{i}=0 \\
\sigma_{\mathrm{k}-1}^{\mathrm{i}} & =\mathrm{SoC}_{\mathrm{k}-1}+\sqrt{(\mathrm{n}+\lambda) \hat{\mathrm{P}}_{\mathrm{SoC}, \mathrm{k}-1}}, \mathrm{i}=1 \sim \mathrm{n} \\
\sigma_{\mathrm{k}-1}^{\mathrm{i}} & =\mathrm{SoC}_{\mathrm{k}-1}-\sqrt{(\mathrm{n}+\lambda) \hat{\mathrm{P}}_{\mathrm{SoC}, \mathrm{k}-1}}, \mathrm{i}=\mathrm{n}+1 \sim 2 \mathrm{n}
\end{aligned}\right.
$$

where $\mathrm{n}$ is the dimension of state vector which means $\mathrm{n}=1 ; \lambda$ is a scaling parameter used to reduce the total prediction error.

$$
\lambda=\alpha^{2}(\mathrm{n}+\kappa)-\mathrm{n}
$$

$\alpha$ controls the distribution state of the sigma points, $k$ needs to satisfy $k \geq 0$. In this study, these two parameters are set as follows: $\alpha=1 ; \kappa=1$.

Weight calculating:

$$
\left\{\begin{array}{l}
\omega_{\mathrm{m}}^{0}=\frac{\lambda}{\mathrm{n}+\lambda} \\
\omega_{\mathrm{c}}^{0}=\frac{\lambda}{\mathrm{n}+\lambda}+\left(1-\alpha^{2}+\beta\right) \\
\omega_{\mathrm{m}}^{\mathrm{i}}=\omega_{\mathrm{c}}^{\mathrm{i}}=\frac{1}{2(\mathrm{n}+\lambda)}, \mathrm{i}=1 \sim 2 \mathrm{n}
\end{array}\right.
$$

where $\beta$ is used to incorporate prior knowledge of the distribution and set to 2 for Gaussian distributions. 
Sigma points one-step prediction:

$$
\sigma_{\mathrm{k} \mid \mathrm{k}-1}^{\mathrm{i}}=\mathrm{F}\left(\sigma_{\mathrm{k}-1}^{\mathrm{i}}, \mathrm{i}_{\mathrm{L}, \mathrm{k}}\right)
$$

Time-update for SoC:

$$
\left\{\begin{array}{l}
\hat{S o C}_{k \mid k-1}=\sum_{i=0}^{2 n} \omega_{m, S o C}^{i} \sigma_{k \mid k-1}^{i} \\
\hat{P}_{S o C, k \mid k-1}=\sum_{i=0}^{2 n} \omega_{c, S o C}^{i}\left[\operatorname{SoC}_{k \mid k-1}-\sigma_{k \mid k-1}^{i}\right]\left[S \hat{C}_{k \mid k-1}-\sigma_{k \mid k-1}^{i}\right]^{T}+Q_{S o C, k-1}
\end{array}\right.
$$

Step 3: Measurement-update for SoC at k-moment

Second sigma point calculating:

$$
\left\{\begin{aligned}
\sigma_{\mathrm{k} \mid \mathrm{k}-1}^{\mathrm{i}} & =\mathrm{SôC}_{\mathrm{k} \mid \mathrm{k}-1}, \mathrm{i}=0 \\
\sigma_{\mathrm{k} \mid \mathrm{k}-1}^{\mathrm{i}} & =\mathrm{SOC}_{\mathrm{k} \mid \mathrm{k}-1}+\sqrt{(\mathrm{n}+\lambda) \hat{\mathrm{P}}_{\mathrm{k} \mid \mathrm{k}-1}}, \mathrm{i}=1 \sim \mathrm{n} \\
\sigma_{\mathrm{k} \mid \mathrm{k}-1}^{\mathrm{i}} & =\operatorname{SoC}_{\mathrm{k} \mid \mathrm{k}-1}-\sqrt{(\mathrm{n}+\lambda) \hat{\mathrm{P}}_{\mathrm{k} \mid \mathrm{k}-1}}, \mathrm{i}=\mathrm{n}+1 \sim 2 \mathrm{n}
\end{aligned}\right.
$$

Sigma point observation prediction:

$$
u_{\mathrm{L}, \mathrm{k} \mid \mathrm{k}-1}^{\mathrm{i}}=\mathrm{G}\left(\sigma_{\mathrm{k} \mid \mathrm{k}-1}^{\mathrm{i}}, \hat{\theta}_{\mathrm{k}-1}\right)
$$

Measurement-update for SoC:

$$
\left\{\begin{array}{l}
\hat{\mathrm{u}}_{\mathrm{L}, \mathrm{k} \mid \mathrm{k}-1}=\sum_{\mathrm{i}=0}^{2 n} \omega_{\mathrm{m}, \mathrm{SoC}}^{\mathrm{i}} \mathrm{u}_{\mathrm{L}, \mathrm{k} \mid \mathrm{k}-1}^{\mathrm{i}} \\
\mathrm{P}_{\mathrm{uu}}=\sum_{\mathrm{i}=0}^{2 n} \omega_{\mathrm{c}, \mathrm{SoC}}^{\mathrm{i}}\left[\hat{\mathrm{u}}_{\mathrm{L}, \mathrm{k} \mid \mathrm{k}-1}-\mathrm{u}_{\mathrm{L}, \mathrm{k} \mid \mathrm{k}-1}^{\mathrm{i}}\right]\left[\hat{\mathrm{u}}_{\mathrm{L}, \mathrm{k} \mid \mathrm{k}-1}-\mathrm{u}_{\mathrm{L}, \mathrm{k} \mid \mathrm{k}-1}^{\mathrm{i}}\right]^{\mathrm{T}}+\mathrm{R}_{\mathrm{k}-1} \\
\mathrm{P}_{\mathrm{SoCu}}=\sum_{\mathrm{i}=0}^{2 n} \omega_{\mathrm{c}, \mathrm{SoC}}^{\mathrm{i}}\left[\mathrm{SoO}_{\mathrm{k} \mid \mathrm{k}-1}-\sigma_{\mathrm{k} \mid \mathrm{k}-1}^{\mathrm{i}}\right]\left[\hat{\mathrm{u}}_{\mathrm{L}, \mathrm{k} \mid \mathrm{k}-1}-\mathrm{u}_{\mathrm{L}, \mathrm{k} \mid \mathrm{k}-1}^{\mathrm{i}}\right]^{\mathrm{T}} \\
\mathrm{K}_{\mathrm{k}}=\mathrm{P}_{\mathrm{SoCu}} \mathrm{P}_{\mathrm{uu}}{ }^{-1} \\
\mathrm{Sô}_{\mathrm{k}}=\hat{S O O}_{\mathrm{k} \mid \mathrm{k}-1}+\mathrm{K}_{\mathrm{k}}\left(\mathrm{u}_{\mathrm{L}, \mathrm{k}}-\hat{\mathrm{u}}_{\mathrm{L}, \mathrm{k} \mid \mathrm{k}-1}\right) \\
\hat{\mathrm{P}}_{\mathrm{SoC}, \mathrm{k}}=\hat{\mathrm{P}}_{\mathrm{SoC}, \mathrm{k} \mid \mathrm{k}-1}-\mathrm{K}_{\mathrm{k}} \mathrm{P}_{\mathrm{uu}} \mathrm{K}_{\mathrm{k}}^{\mathrm{T}}
\end{array}\right.
$$

Noise-update for SoC:

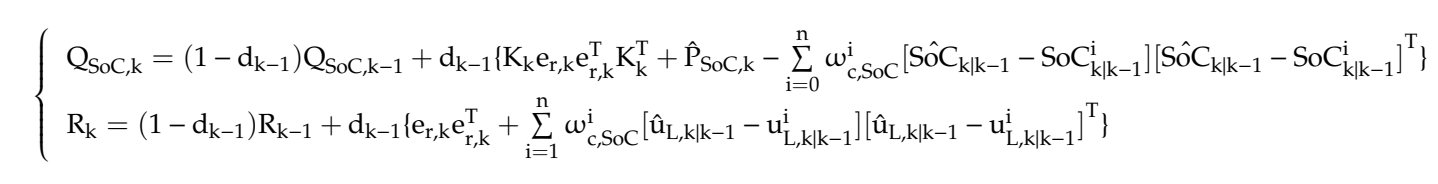

where $e_{r, k}$ represent the error of prediction at k-moment, $u_{L, k}$ is the experimental data of terminal voltage at $\mathrm{k}$-moment. $\mathrm{b}$ is the forgetting factor which is used to reduce the influence of historical data and set to 0.99 in this study.

$$
\begin{aligned}
& \mathrm{e}_{\mathrm{r}, \mathrm{k}}=\left|\mathrm{u}_{\mathrm{L}, \mathrm{k}}-\hat{\mathrm{u}}_{\mathrm{L}, \mathrm{k} \mid \mathrm{k}-1}\right| \\
& \mathrm{d}_{\mathrm{k}}=(1-\mathrm{b}) /\left(1-\mathrm{b}^{\mathrm{k}}\right)
\end{aligned}
$$

Step 4: Judgement for $\mathrm{e}_{\mathrm{r}, \mathrm{k}}$ :

if $\mathrm{e}_{\mathrm{r}, \mathrm{k}}>\sigma$, then:

$$
\hat{\theta}_{\mathrm{k}}=\hat{\theta}_{\mathrm{k}-1}
$$

which means to keep the parameters still and get back to step 2;

Else, open the parameter adjuster and continue to Step 5:

Step 5: Time-update for $\theta$ at k-moment which is similar with Equations (13)-(17). 
Step 6: Measurement-update for $\theta$ at k-moment which is similar with Equations (18)-(20).

After that, get back to Step 2 for the next moment.

\section{Experiments}

\subsection{Experimental Bench}

In this study, we chose one specific manufacturer's $\mathrm{LiNi}_{\mathrm{x}} \mathrm{Mn}_{\mathrm{y}} \mathrm{Co}_{1-\mathrm{x}-\mathrm{y}} \mathrm{O}_{2}$ lithium-ion battery as the experimental object considering not only actual condition but also it is a lithium-ion battery with typical ternary cathode material, and its basic parameters are shown in Table 1. Neware (Shen Zhen, China) Battery Test System CT-4008-5V100A was selected as battery testing equipment, and a PC host was equipped to control the testing step and record the experimental data. Qin Zhuo Huan Ce (Dong Guan, China) thermal chamber GDW-100 was chosen as ambient temperature controller. Since this study did not involve the influence of ambient temperature of batteries, all experiments were conducted at $25^{\circ} \mathrm{C}$. The structure of the experimental bench is shown in Figure 5.

Table 1. Parameters of the experimental battery.

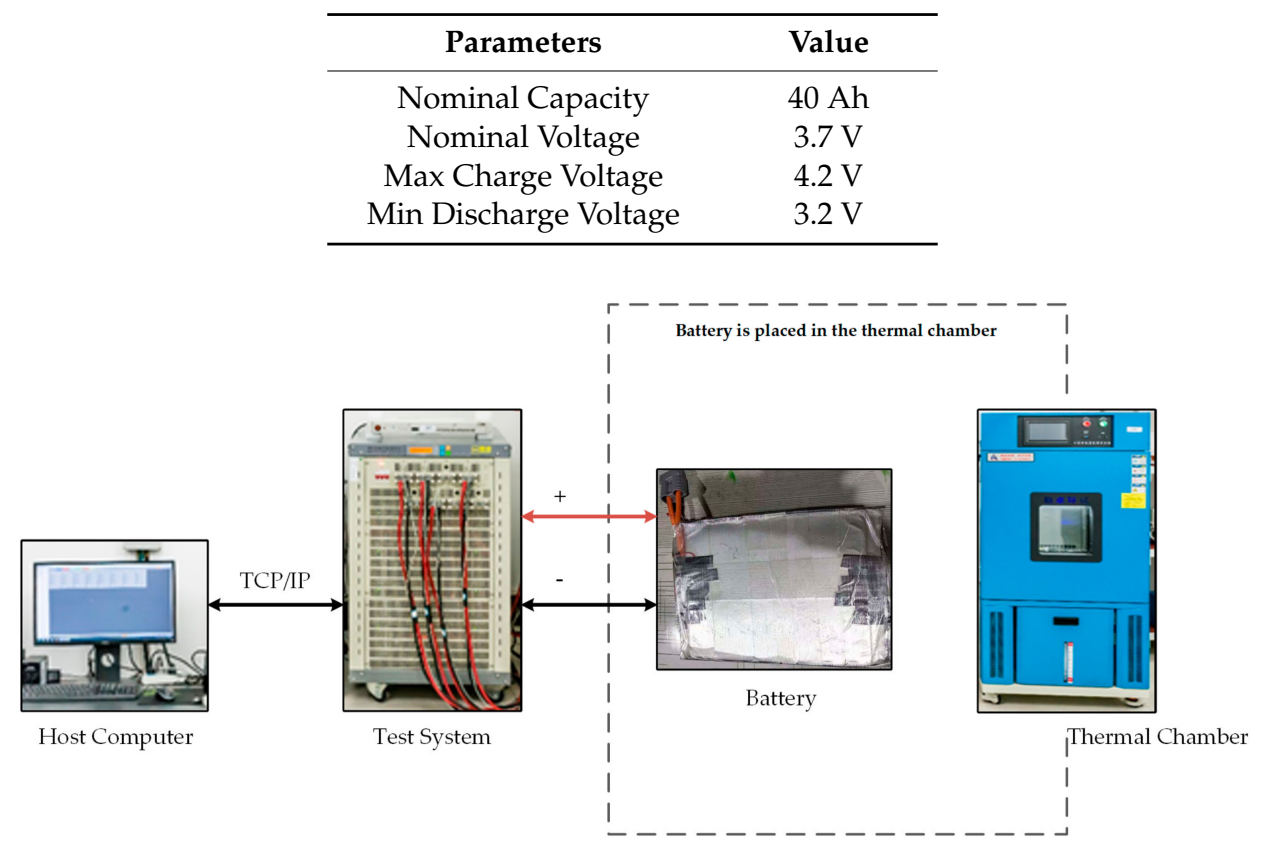

Figure 5. Structure of experimental bench.

\subsection{Experimental Methods}

In this study, HPPC test was designed to identify the $\mathrm{u}_{\mathrm{oc}}-\mathrm{SoC}$ relationship and the $\mathrm{RC}$ parameters of battery model simultaneously. A cycle consists of a $1 / 3 \mathrm{C}$ constant current discharge, a $3 \mathrm{~h}$ recovery and a pair of $1 \mathrm{C}$ pulse charge and discharge. The specific current and voltage curves are shown in Figure 6. The experimental results can also be used to verify the model and algorithm as an operating condition.

In order to further verify the performance of the proposed algorithm, the Dynamic Stress Test (DST) was carried out, refer to [26]. The DST is to simulate the dynamic power of driving electric vehicle by loading preset current, so as to test the dynamic performance of the battery. The experimental curves of current and voltage in one cycle are shown in Figure 7. 

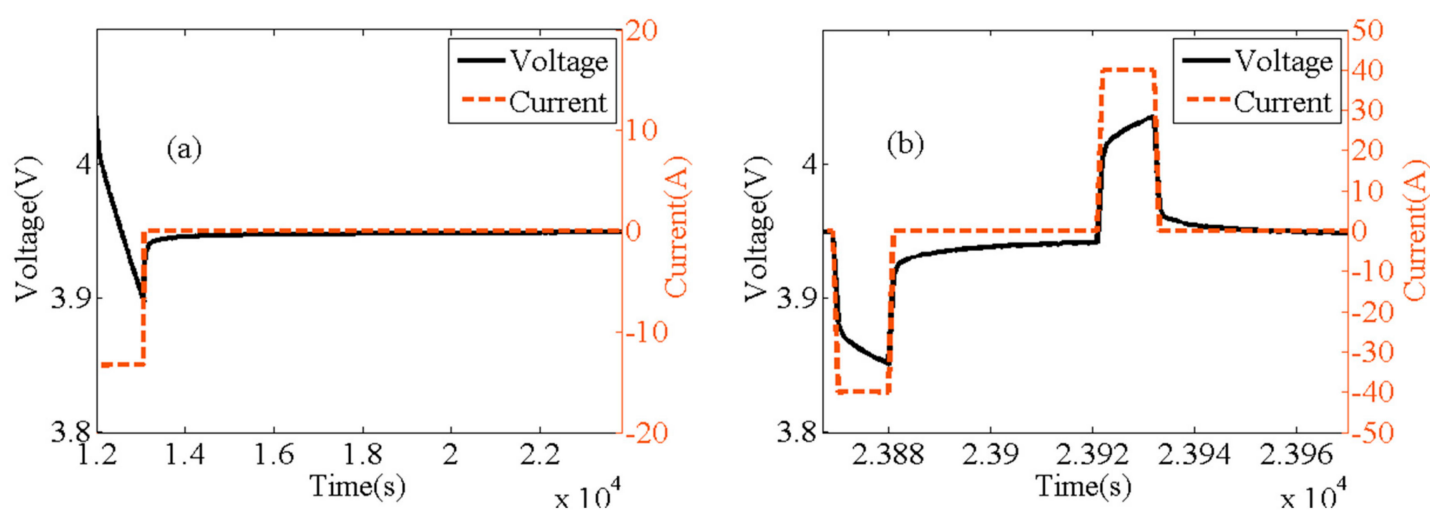

Figure 6. (a) The experimental curve of voltage and current of $1 / 3 \mathrm{C}$ discharge and $3 \mathrm{~h}$ rest in a cycle under Hybrid Pulse Power Characterization (HPPC) test. (b) The experimental curve of voltage and current of $1 \mathrm{C}$ pulse discharge and charge in a cycle under HPPC test.

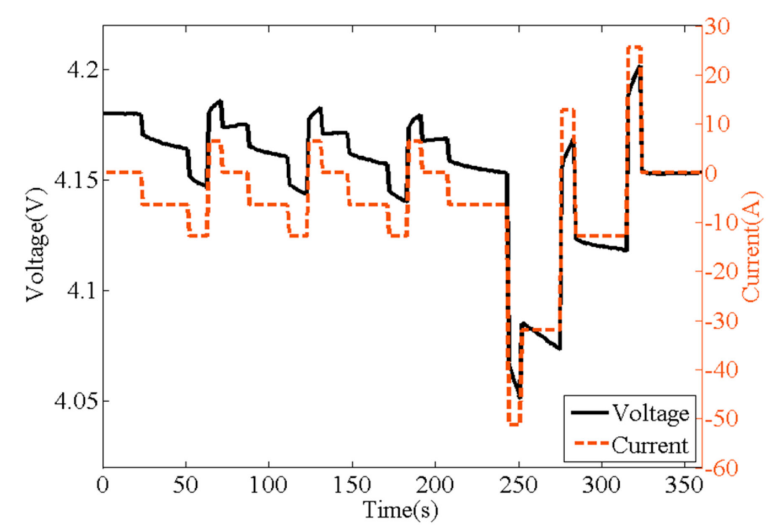

Figure 7. The experimental curve of voltage and current in a cycle under DST test.

\section{Results and Discussion}

\subsection{Off-Line Parameters Identification Results}

The off-line parameter identification method described in Section 2 and the experimental method described in Section 4 are used to identify the $\mathrm{u}_{\mathrm{oc}}$-SoC relationship and RC parameters in the model. This was done according to the data collecting method described in Section 2 and fitting curve function referred to in Equation (3). The data scatters and their fitting curve are shown in Figure 8. The constants of the fitting curve function (six-order polynomial) $\mathrm{K}_{\mathrm{i}}$ are listed in Table 2. RC parameters can be divided into identification results at pulse discharge or at long discharge recovery, as shown in Table 3. It can be found that even for the same SoC stage, the identification results of $\tau_{1}$ and $\tau_{2}$ at the pulse discharge are quite different from those at the long discharge recovery. Taking SoC $=0.902$ as an example, the pulse discharge recovery curve and the long discharge recovery curve in the first $40 \mathrm{~s}$ are compared in Figure 9. It can be considered that the recovery speed of the pulse discharge is faster than that of the long discharge, which indicates that SoC is not the only factor affecting the dynamic change of RC parameters. The $\mathrm{u}_{\mathrm{oc}}$-SoC relationship can be considered stable within a certain number of cycles [27]. Therefore, RC parameters have more complex dynamic processes in the operation of batteries, and more easily affect the accuracy of the model. 


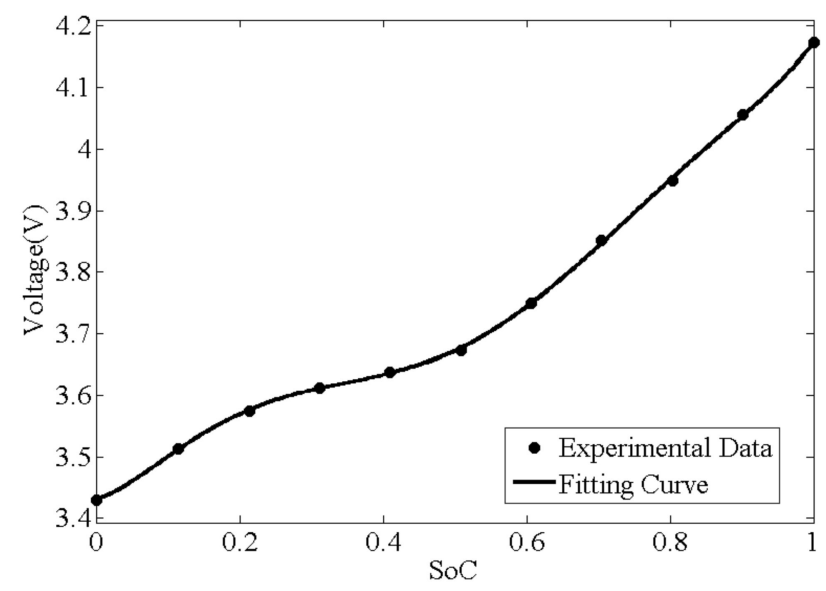

Figure 8. $\mathrm{u}_{\mathrm{oc}}$-State-Of-Charge $(\mathrm{SoC})$ fitting curve.

Table 2. Identification results of the fitting curve.

\begin{tabular}{cccccccc}
\hline Parameters & $\mathbf{K}_{\mathbf{0}}$ & $\mathbf{K}_{\mathbf{1}}$ & $\mathbf{K}_{\mathbf{2}}$ & $\mathbf{K}_{\mathbf{3}}$ & $\mathbf{K}_{\mathbf{4}}$ & $\mathbf{K}_{\mathbf{5}}$ & $\mathbf{K}_{\mathbf{6}}$ \\
\hline Value & 3.43 & 0.3725 & 5.862 & -32.58 & 68.02 & -60.73 & 19.8 \\
\hline
\end{tabular}

Table 3. Identification results of RC parameters.

\begin{tabular}{|c|c|c|c|c|c|c|c|c|}
\hline \multirow{2}{*}{\multicolumn{2}{|c|}{$\begin{array}{c}\text { Identification Area } \\
\text { Parameters }\end{array}$}} & \multicolumn{2}{|c|}{ Long Discharge Recovery } & \multicolumn{5}{|c|}{ Pulse Discharge } \\
\hline & & $\tau_{1}(\mathrm{~s})$ & $\tau_{2}(\mathrm{~s})$ & $\tau_{1}(\mathrm{~s})$ & $\tau_{2}(\mathrm{~s})$ & $\mathrm{R}_{1}(\Omega)$ & $\mathrm{R}_{2}(\Omega)$ & $\mathrm{R}_{0}(\Omega)$ \\
\hline \multirow{9}{*}{ SoC $=$} & 0.902 & 1613 & 79.94 & 16.13 & 1.001 & 0.0010 & 0.000278 & 0.0017 \\
\hline & 0.834 & 2695 & 150.22 & 14.13 & 0.741 & 0.0011 & 0.000227 & 0.0017 \\
\hline & 0.705 & 2045 & 274.57 & 12.70 & 0.640 & 0.0011 & 0.000218 & 0.0017 \\
\hline & 0.607 & 2149 & 126.33 & 15.40 & 0.831 & 0.0011 & 0.000239 & 0.0017 \\
\hline & 0.508 & 2838 & 193.57 & 14.18 & 0.765 & 0.0008 & 0.000211 & 0.0017 \\
\hline & 0.410 & 2973 & 268.96 & 12.20 & 0.582 & 0.0007 & 0.000205 & 0.0017 \\
\hline & 0.312 & 2458 & 327.44 & 12.70 & 0.589 & 0.0008 & 0.000249 & 0.0018 \\
\hline & 0.213 & 2670 & 332.12 & 11.51 & 0.468 & 0.0008 & 0.000346 & 0.0018 \\
\hline & 0.115 & 4390 & 414.77 & 11.84 & 0442 & 0.0012 & 0.000613 & 0.0018 \\
\hline
\end{tabular}

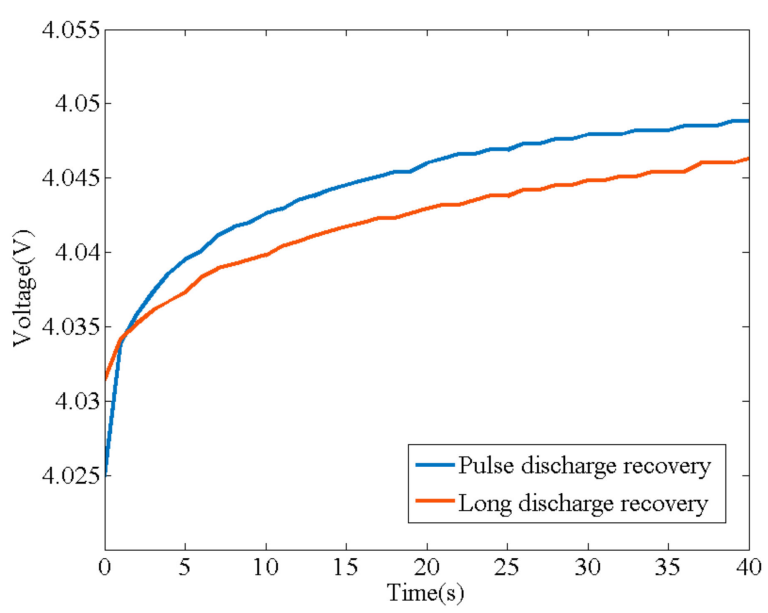

Figure 9. The comparison of recovery voltage curve between pulse discharge and long discharge when SoC $=0.902$ (first $40 \mathrm{~s})$.

\subsection{Advance Explanation of Model Accuracy Evaluation Method}

It is worth discussing that the model parameters obtained from the off-line identification are input into the open-loop battery model in Equation (8) and a common UKF for SoC estimation based 
on this model. The predicted voltage results are quite different. Taking the SoC drops from 0.902 to 0.834 in HPPC test as an example, the RC parameters are taken as the values when $\mathrm{SoC}=0.902$. Figure 10a is the curve comparison of the output voltage of the open-loop model, the predicted voltage of the UKF and the experimental data; Figure $10 \mathrm{~b}$ is the error comparison. It can be seen that the predicted voltage error of UKF is significantly smaller than that of open-loop model even though the parameters adopted are identical. One of the characteristics of Kalman filter is that it can modify the state according to the observation (in this case, the experimental voltage data) to get its maximum likelihood estimation. This determines that the algorithm can gradually correct the initial value of the state when it is inaccurate, which also means the prediction can always converge to the observation [23]. However, in the case of inaccurate model, the negative impact of this feature is that the filter may over correct the state and reduce its estimation accuracy, which will be verified in 5.3. Therefore, the error between the voltage prediction of closed-loop algorithm and the experimental data cannot reflect the actual model accuracy, on the contrary, it may confuse the judgement. In this study, the modified parameters of all the algorithms in 5.3 will be input into the open-loop battery model, and the effects of model accuracy on SoC estimation are compared.
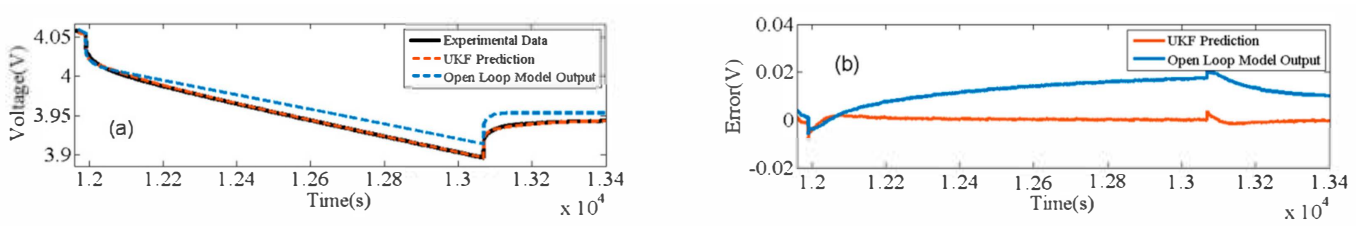

Figure 10. (a) The voltage comparison between experimental data, UKF prediction and open loop model output when SoC drops from 0.902 to 0.834 . (b) The error comparison between UKF prediction and open loop model output when SoC drops from 0.902 to 0.834 .

\subsection{Comparative Analysis of Algorithms}

The structure of the algorithm comparison experiment in this section is shown in Figure 11. For each algorithm, the experimental current $i_{L, k}$ and voltage $Y_{k}$ are loaded, the SoC estimation curves and the algorithm predicted voltage curves are drawn. The dynamic parameters modified by the algorithms are input into the open-loop model in Equation (8), and the output voltage curves are drawn.

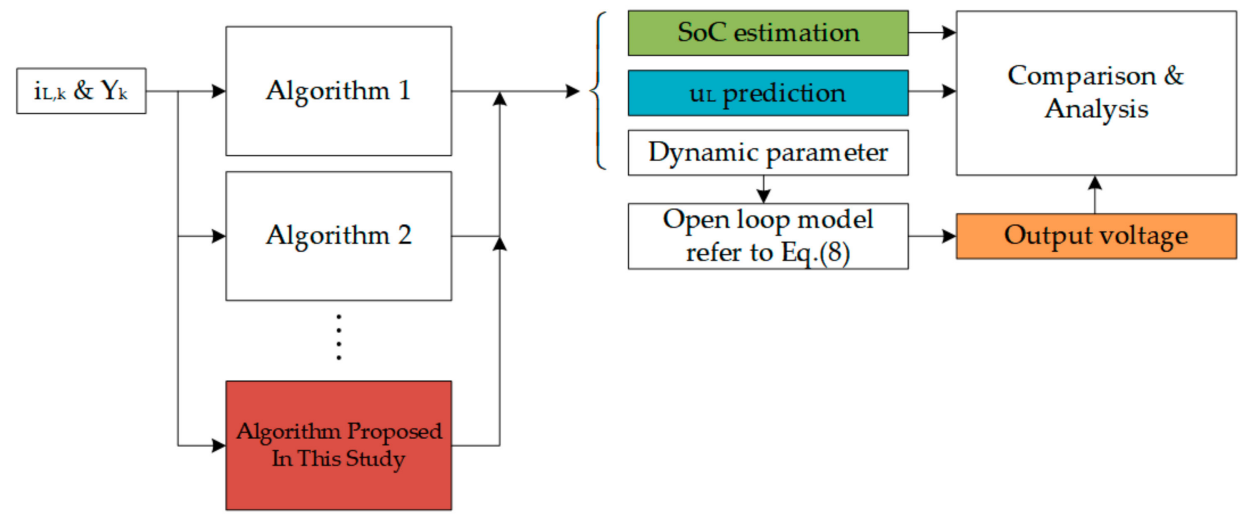

Figure 11. Structure of algorithms comparison experiment.

\subsubsection{Comparison of SoC Estimation and Parameter Modification Accuracy}

In order to analyze the SoC estimation and parameter modification accuracy of the traditional AUKF-UKF and the proposed state-parameters separated AUKF-UKF, this section compares these two algorithms with an AUKF. In HPPC test, the initial SoC of these algorithms are set to 0.902 (reference initial value is 0.902); in DST test, the initial SoC are set to 1 (reference initial value 
is 1). The initial parameters under both tests are set to the identification result of pulse discharge at $\mathrm{SoC}=0.902$ in HPPC test.

Figure 12a is the SoC estimation result of three algorithms under HPPC test, and Figure 12b is the SoC estimation error of three algorithms. The black curve represents the reference SoC based on the experimental measurement of its initial value and ampere-time counting method combined with the current data; the red curve, the blue curve and the yellow curve represent AUKF, state-parameter unseparated AUKF-UKF, and state-parameter separated AUKF-UKF respectively. Similarly, Figure 13 shows the $u_{\mathrm{L}}$ prediction results of three algorithms under HPPC test and their errors between experimental voltage data. The output voltage shown in Figure 14a is obtained by inputting the parameters which are modified by the three algorithms (AUKF's parameters are constant) into the open-loop model. Figure $14 \mathrm{~b}$ shows their errors between experimental voltage data. error $_{\max }$ (maximum error) and root mean square error (RMSE) are set to analyze the error data in this study. The calculation formula is shown in Equation (25).

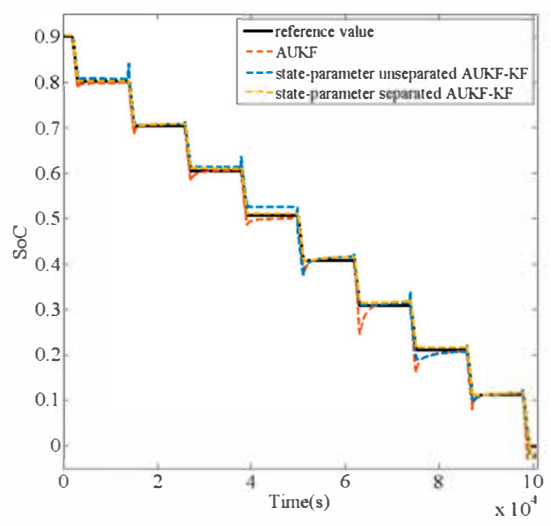

(a)

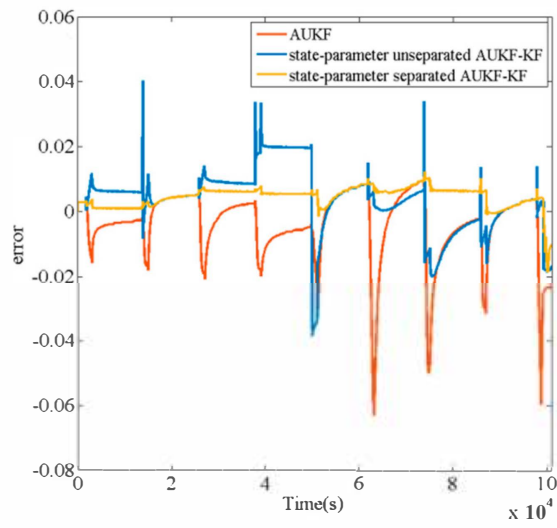

(b)

Figure 12. (a) The comparison of the SoC estimation between three algorithms under HPPC test; (b) The comparison of the SoC estimation error between three algorithms under HPPC test.
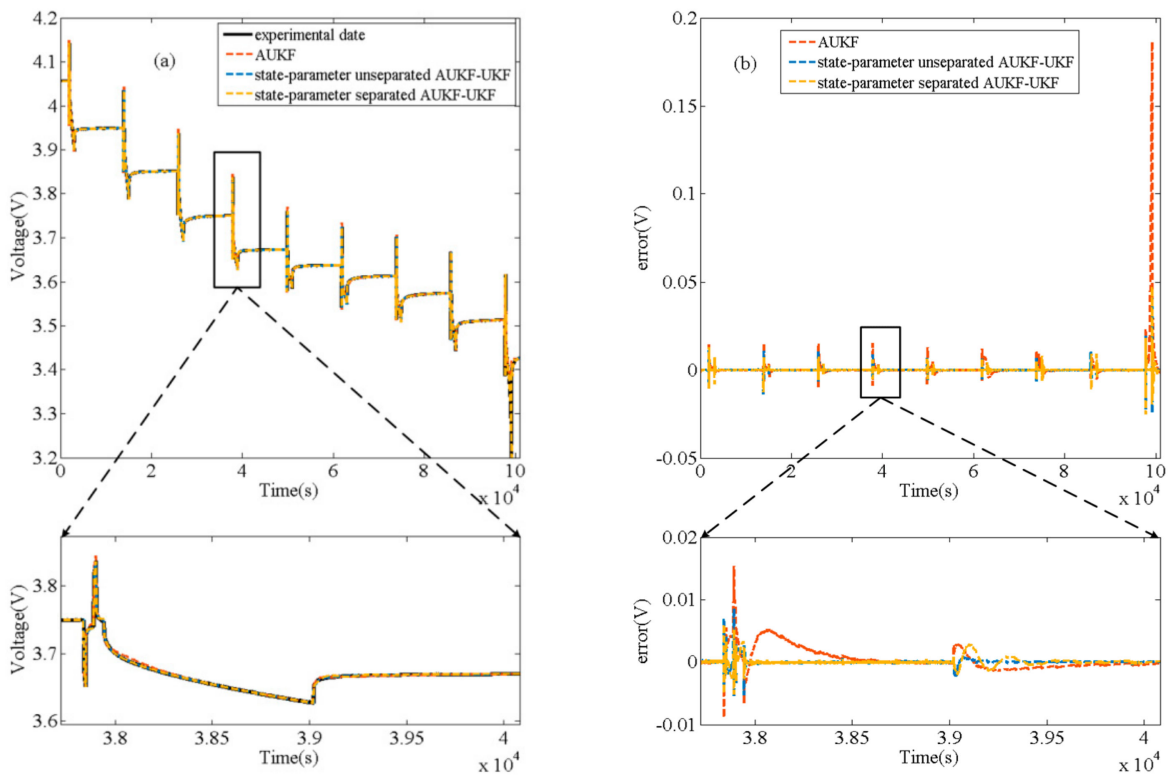

Figure 13. (a) The comparison of the $\mathrm{u}_{\mathrm{L}}$ prediction between three algorithms under HPPC test; (b) The comparison of the $\mathrm{u}_{\mathrm{L}}$ prediction error between three algorithms under HPPC test. 


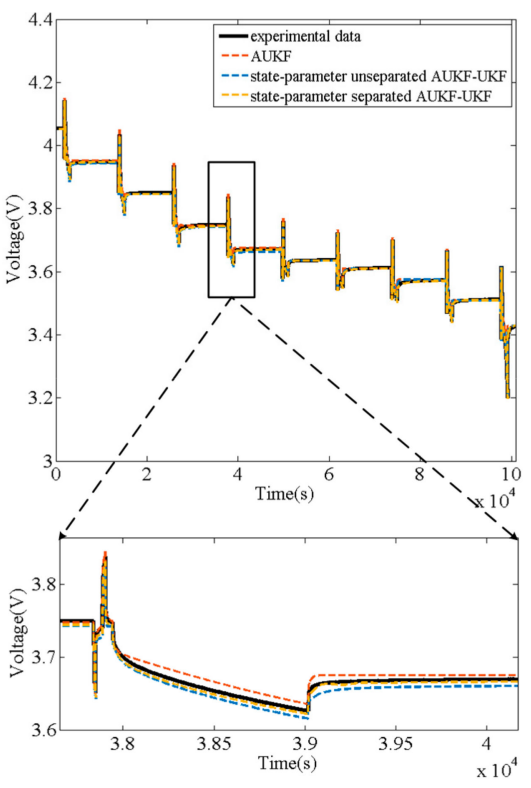

(a)

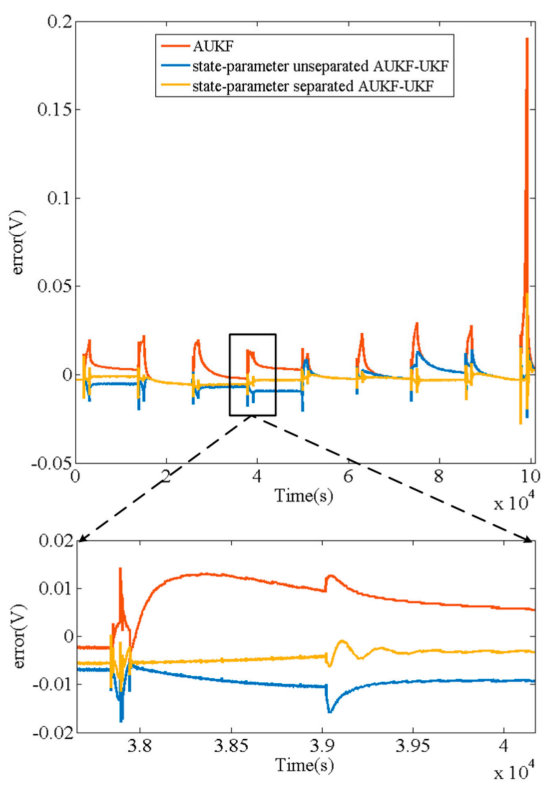

(b)

Figure 14. (a) The comparison of the open-loop model output between three algorithms under HPPC test; (b) The comparison of the open-loop model output error between three algorithms under HPPC test.

Where $X_{k}$ is the estimation of k-moment and $X^{r e f}{ }_{k}$ is the reference of k-moment. All calculation results of the above two parameters under HPPC test are shown in Table 4.

Table 4. Error analysis of different algorithms under HPPC test.

\begin{tabular}{cccc}
\hline Algorithms & Items & Error $_{\text {max }}$ & RMSE \\
\hline \multirow{3}{*}{ AUKF } & SoC estimation & 0.0631 & 0.0122 \\
& u $_{\text {L prediction }}$ & $0.1865 \mathrm{~V}$ & $0.0066 \mathrm{~V}$ \\
& Open loop model output & $0.1904 \mathrm{~V}$ & $0.0097 \mathrm{~V}$ \\
\hline \multirow{3}{*}{ state-parameter unseparated AUKF-UKF } & SoC estimation & 0.0404 & 0.00104 \\
& u $_{\text {L prediction }}$ & $0.0403 \mathrm{~V}$ & $0.00049 \mathrm{~V}$ \\
& Open loop model output & $0.0405 \mathrm{~V}$ & $0.0056 \mathrm{~V}$ \\
\hline \multirow{3}{*}{ state-parameter separated AUKF-UKF } & SoC estimation & 0.0187 & 0.00056 \\
& Open loop model output & $0.0474 \mathrm{~V}$ & $0.00092 \mathrm{~V}$ \\
& & & $0.0034 \mathrm{~V}$ \\
\hline
\end{tabular}

$$
\left\{\begin{array}{l}
\text { error }_{\max }=\operatorname{Max}\left|X_{\mathrm{k}}-X_{\mathrm{k}}^{\mathrm{ref}}\right| \\
\mathrm{RMSE}=\sqrt{\frac{1}{\mathrm{n}} \sum_{\mathrm{k}=1}^{\mathrm{n}}\left(X_{\mathrm{k}}-X_{\mathrm{k}}^{\mathrm{ref}}\right)^{2}}
\end{array}\right.
$$

From Figures 12-14 and Table 4, it can be found that the error ${ }_{\max }$ and RMSE of the $\mathrm{u}_{\mathrm{L}}$ predictions of state-parameter unseparated AUKF-UKF are smaller than those of the state-parameter separated AUKF-UKF. If the model accuracy is referenced by this result, the conclusion is that the traditional AUKF-UKF has better parameter modification performance, and should have higher SoC estimation accuracy. However, the experimental results show that the state-parameter separated AUKF-UKF has higher SoC estimation accuracy than the other two algorithms. The point of view in 5.2 is verified here that the error between the predicted terminal voltage of the filter and the experimental voltage data is unreliable as a criterion to measure the accuracy of the model. The reason is that the closed-loop algorithm corrects the state based on the observation and can also converge the predicted terminal 
voltage to the experimental data when the model parameters are inaccurate. Thus, the state is over correct and the error has increased. According to 5.2, by comparing the terminal voltage output after inputting the dynamic parameters of the three algorithms to the open-loop model, we find that the error AUKF > state-parameter unseparated AUKF-UKF > state-parameter separated AUKF-UKF, which is consistent with the comparison results of SoC estimation. It shows that the proposed state-parameter separated AUKF-UKF has higher SoC estimation and parameter modification accuracy.

Similarly, Figure 15 shows SoC estimation and errors of three algorithms under DST test; Figure 16 shows $\mathrm{u}_{\mathrm{L}}$ prediction and errors of three algorithms under DST test. The output voltage and error shown in Figure 17 is obtained by inputting the parameters which are modified by the three algorithms (AUKF's parameters are constant) into the open-loop model. Red curve, blue curve and yellow curve represent AUKF, state-parameter unseparated AUKF-UKF and state-parameter separated AUKF-UKF respectively. The analysis results of the error data are shown in Table 5. The results show that the $\mathrm{u}_{\mathrm{L}}$ prediction error of state-parameter separated AUKF-UKF is larger than that of traditional AUKF-UKF, while the output voltage error of open-loop model is smaller, the estimation accuracy of SoC is higher. Therefore, compared with the other two algorithms, state-parameter separated AUKF-UKF has higher SoC estimation accuracy and better parameter modification performance under DST test.

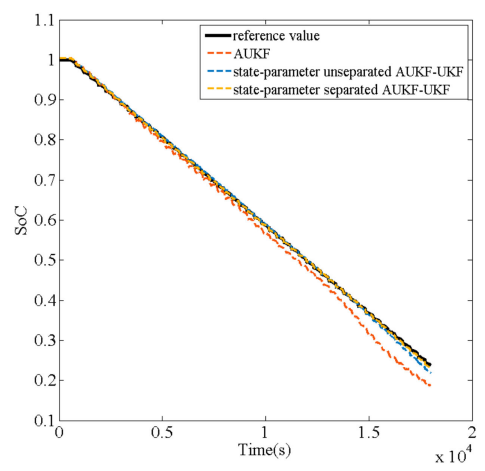

(a)

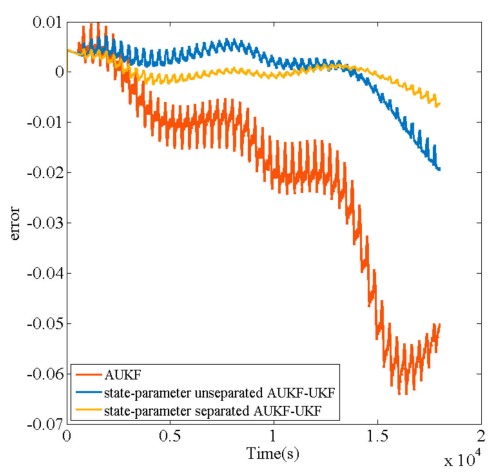

(b)

Figure 15. (a) The comparison of the SoC estimation between three algorithms under DST test; (b) The comparison of the SoC estimation error between three algorithms under DST test.

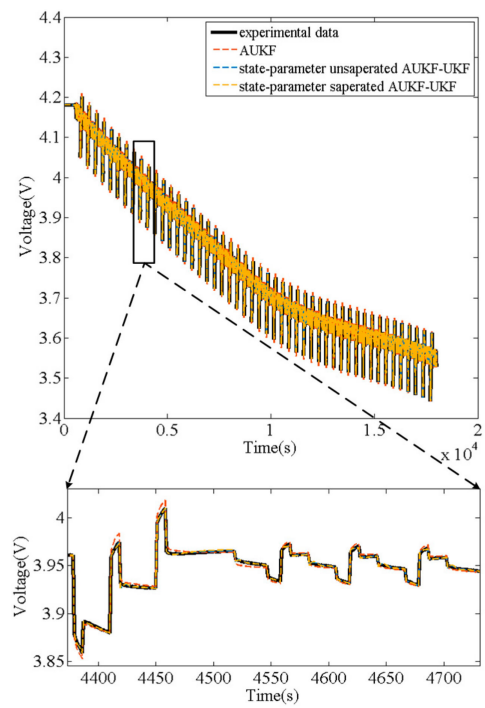

(a)

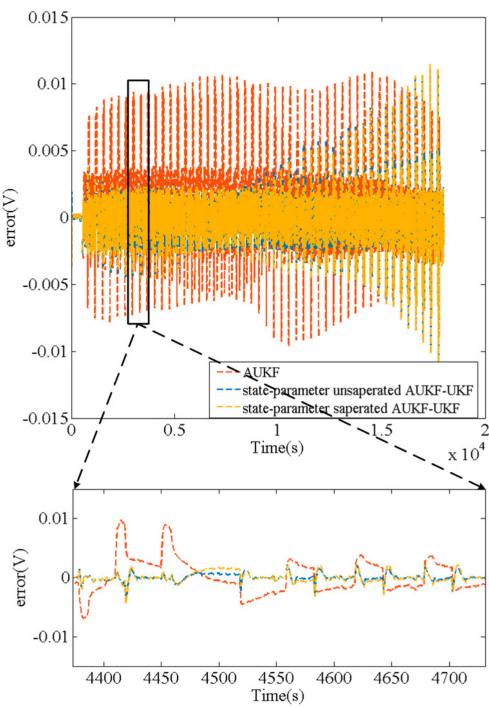

(b)

Figure 16. (a) The comparison of the $\mathrm{u}_{\mathrm{L}}$ prediction between three algorithms under DST test; (b) The comparison of the $\mathrm{u}_{\mathrm{L}}$ prediction error between three algorithms under DST test. 


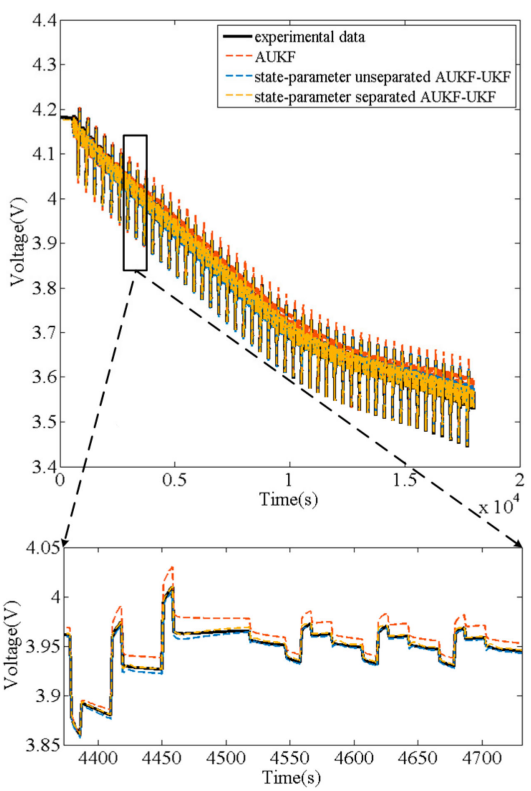

(a)

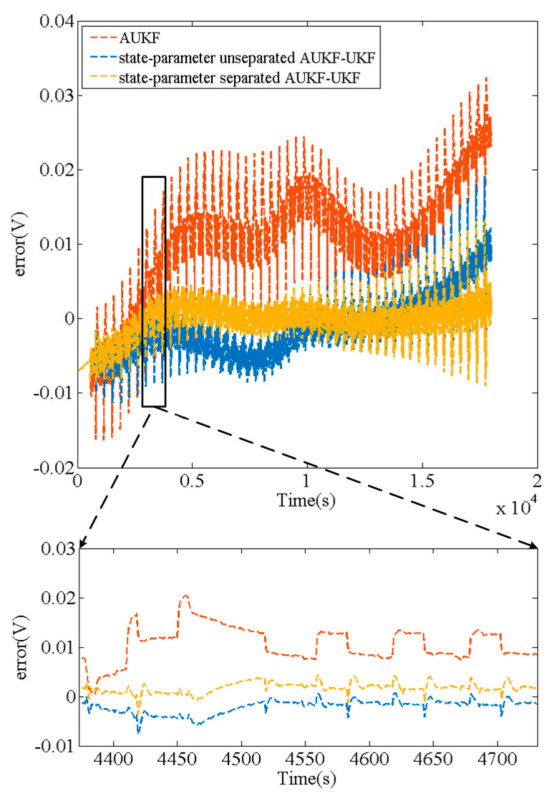

(b)

Figure 17. (a) The comparison of the open-loop output between three algorithms under DST test; (b) The comparison of the open-loop output error between three algorithms under DST test.

Table 5. Error analysis of different algorithms under DST test.

\begin{tabular}{cccc}
\hline Algorithms & Items & Error $_{\text {max }}$ & RMSE \\
\hline \multirow{3}{*}{ AUKF } & SoC estimation & 0.0642 & 0.0279 \\
& u $_{\text {L prediction }}$ & $0.0109 \mathrm{~V}$ & $0.0031 \mathrm{~V}$ \\
& Open loop model output & $0.0323 \mathrm{~V}$ & $0.0129 \mathrm{~V}$ \\
\hline \multirow{3}{*}{ State-parameter unseparated AUKF-UKF } & SoC estimation & 0.0195 & 0.00059 \\
& u $_{\text {L prediction }}$ & $0.0109 \mathrm{~V}$ & $0.0008 \mathrm{~V}$ \\
& Open loop model output & $0.0191 \mathrm{~V}$ & $0.00041 \mathrm{~V}$ \\
\hline \multirow{3}{*}{ State-parameter separated AUKF-UKF } & SoC estimation & 0.0069 & 0.00021 \\
& upen loop model output & $0.0131 \mathrm{~V}$ & $0.00022 \mathrm{~V}$ \\
\hline
\end{tabular}

\subsubsection{Comparison of Convergence Performance}

According to Section 3.2, the convergence performance of the traditional DUKF is weak when the initial value of the state is inaccurate. The IAUKF-UKF proposed in this paper ranked the two filters to turn the parameter modification on only when the voltage prediction converges to the observed value. In order to verify the reliability of the proposed algorithm, different initial values of SoC (the reference is [1]) are set in this section. The convergence performance of AUKF-UKF and IAUKF-UKF are compared under DST test. In addition, in order to maintain consistency, the two algorithms adopt the state-parameter separated AUKF-UKF proposed in this study.

Figure 18 shows the SoC estimation and $\mathrm{u}_{\mathrm{L}}$ prediction curve of IAUKF-UKF algorithm under different initial SoC. It can be seen that the algorithm can converge to the real value when the initial $\mathrm{SoC}$ is inaccurate. It can also be found that the convergence time increases with the increase of initial error from Table 6. Figure 19 shows model parameter modification curves when the initial SoC is set to 0.7 . It can be found that the parameters are not modified before $1023 \mathrm{~s}$ and remain constant. After $1023 \mathrm{~s}$, the SoC and $\mathrm{u}_{\mathrm{L}}$ prediction have converged to the real value and the parameter modifier is turned on. Figure 20 shows the SoC estimation and $u_{\mathrm{L}}$ prediction curve of the traditional AUKF-UKF under different initial SoC. It can be seen that when the initial SoC is inaccurate, the $u_{L}$ prediction of the AUKF-UKF can still converge to the experimental data, but the SoC estimation has not converged to the 
reference, which means invalid. This shows that it is unreliable to use the convergence performance of $u_{L}$ prediction to judge that of SoC. In summary, the IAUKF-UKF algorithm proposed in this study can overcome the shortcomings of the traditional DUKF in the case of inaccurate initial state, and enhance the robustness of the algorithm.

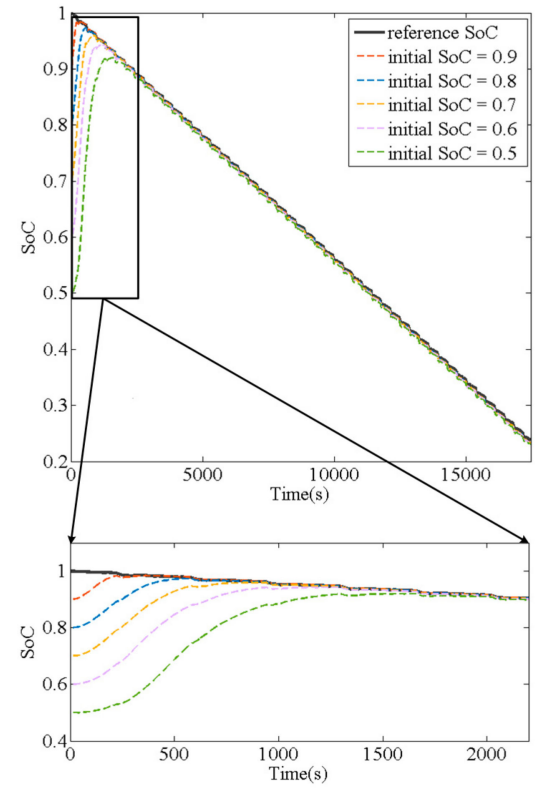

(a)

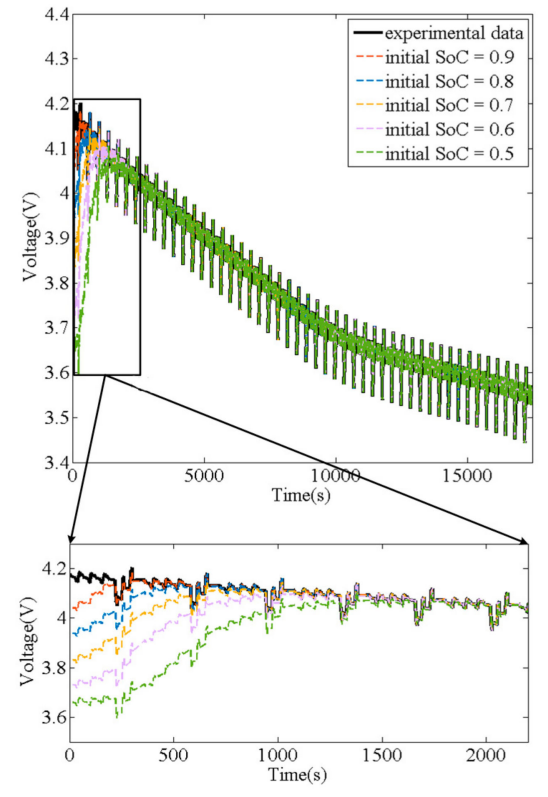

(b)

Figure 18. (a) The comparison of the convergence performance of SoC estimation between different initial value of SoC using IAUKF-UKF under DST test; (b) The comparison of the convergence performance of $u_{\mathrm{L}}$ prediction between different initial value of SoC using IAUKF-UKF under DST test.

Table 6. Convergence time of different initial SoC under DST test.

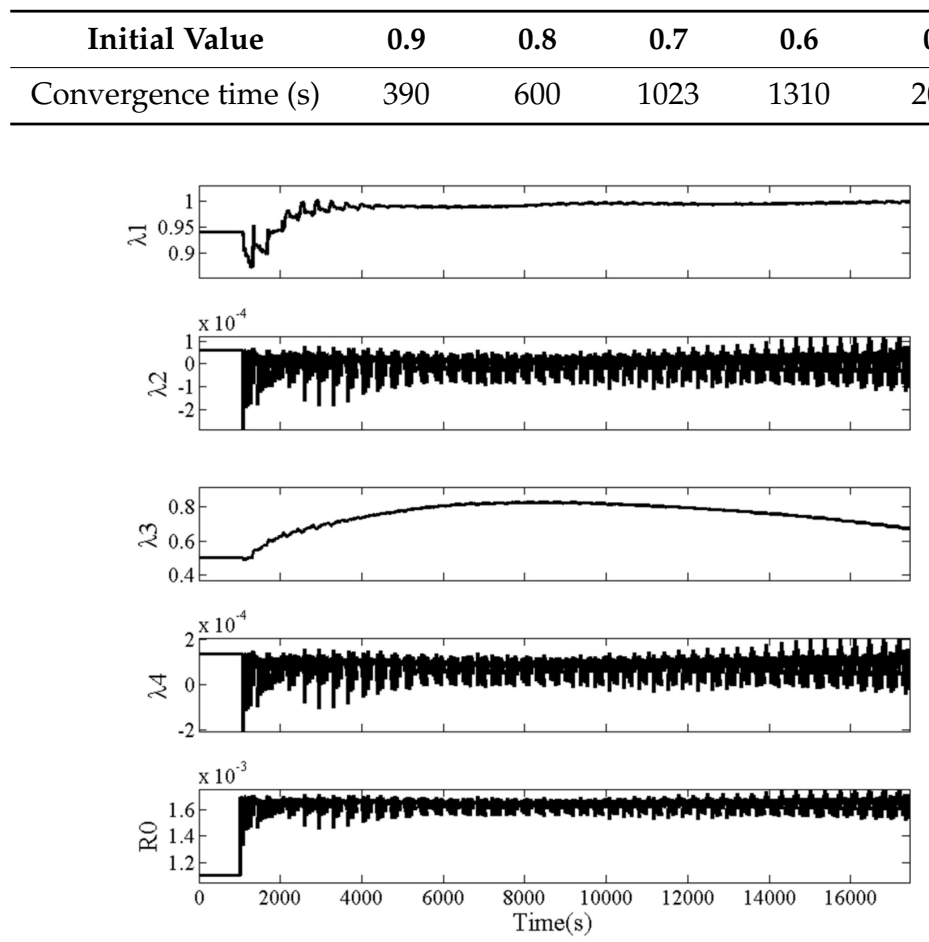

Figure 19. The curves of parameter modification when the initial $\mathrm{SoC}=0.7$. 


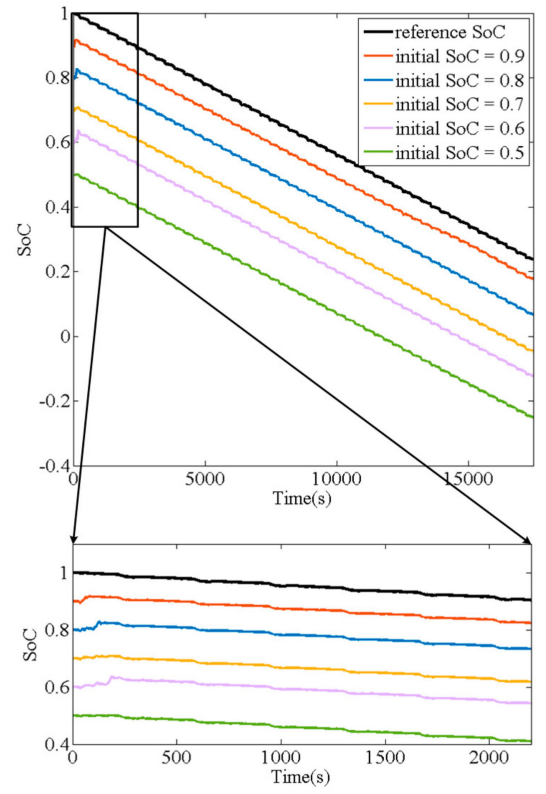

(a)

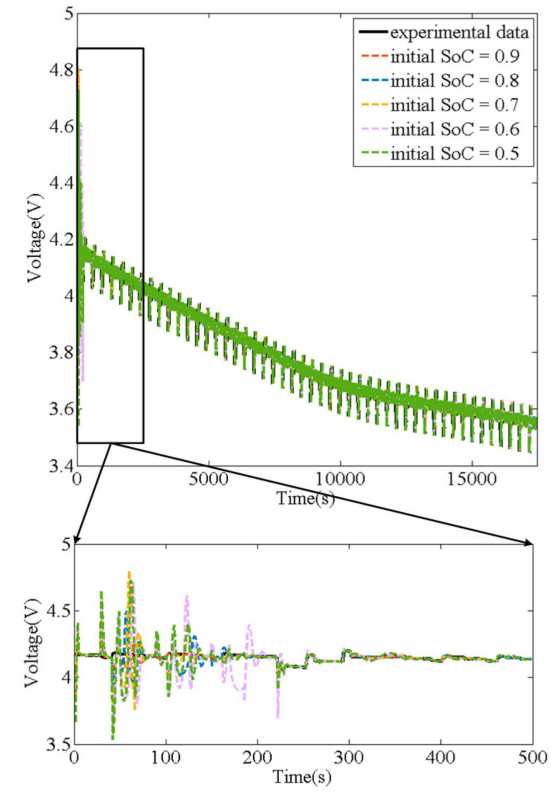

(b)

Figure 20. (a) The comparison of the convergence performance of SoC estimation between different initial value of SoC using AUKF-UKF under DST test; (b) The comparison of the convergence performance of $\mathrm{u}_{\mathrm{L}}$ prediction between different initial value of SoC using AUKF-UKF under DST test.

\section{Conclusions}

In this study, an improved AUKF-UKF based on state-parameters separation is proposed. Its main characteristics are as follows: (1) SoC is the only state variable in the dynamic equation of the model, and the RC parameters of the model are treated as dynamic ones to be modified, which eliminates the strong correlation between state and parameters; (2) The state estimator AUKF and the parameter modifier UKF are treated with different ranks, so that the parameter modifier works only when the terminal voltage prediction of the state estimator converges to the observed value.

First, the proposed state-parameter separated AUKF-UKF is compared with single AUKF and traditional AUKF-UKF. HPPC and DST test are loaded on the three algorithms respectively. Experiment shows that state-parameter separated AUKF-UKF has higher SoC estimation and parameter modification accuracy than the other two algorithms. It is proved that it is unreliable to measure the accuracy of parameter modification by using the error of terminal voltage prediction, while the error of terminal voltage output obtained by inputting dynamic parameters into the open-loop model can reflect the actual situation.

Second, the convergence performance of the proposed IAUKF-UKF and AUKF-UKF under DST test with inaccurate initial SoC is compared. The experimental results show that the proposed IAUKF-UKF can converge the SoC to the reference when the initial value is inaccurate, and the convergence time increases with the increase of the initial error. Traditional AUKF-UKF can converge the terminal voltage prediction to the experimental data, but the misconvergence of SoC makes the estimation invalid. This shows that the convergence performance of terminal voltage prediction is independent from that of the state.

Author Contributions: Conceptualization, C.-X.Y. and Y.-M.X.; methodology, Y.-M.X.; software, Y.-M.X.; validation, Y.-M.X., Z.-Y.S., S.-Y.Y. and R.H.; formal analysis, C.-X.Y.; investigation, C.-X.Y. and Y.-M.X.; resources, C.-X.Y.; data curation, Z.-Y.S., S.-Y.Y. and R.H.; Writing-Original draft preparation, Y.-M.X.; Writing-Review and editing, C.-X.Y. and Y.-M.X.; visualization, Y.-M.X.; supervision, C.-X.Y.; project administration, C.-X.Y.

Funding: This research received no external funding. 
Acknowledgments: The experimental equipment is supported by the Vehicle Power System Lab of School of Automotive Engineering, Chongqing University and Vehicle Inspection Research Institute of Chongqing. The experimental batteries are supported by Chongqing Baizhuan Technology Co., Ltd., Chongqing, China.

Conflicts of Interest: The authors declare no conflict of interest.

\section{References}

1. Huangfu, Y.; Xu, J.; Zhao, D.; Liu, Y.; Gao, F. A novel battery state of charge estimation method based on a super-twisting sliding mode observer. Energies 2018, 11, 1211. [CrossRef]

2. Hannan, M.A.; Xu, J.; Lipu, M.S.H.; Hussian, A.; Mohamed, A. A review of lithium-ion battery state of charge estimation and management system in electric vehicle applications: Challenges and recommendations. Renew. Sustain. Energy Energies 2017, 78, 834-854. [CrossRef]

3. Patrick, J.; Michael, V.; Daniel, R.; David, V.; Werner, J.; Jürgen, G. An electrochemical impedance spectroscopy study on a lithium sulfur pouch cell. Adv. Radio Sci. 2016, 14, 55-62.

4. Stroe, D.I.; Knap, V.; Swierczynski, M.; Stanciu, T.; Schaltz, E.; Teodorescu, R. An electrochemical impedance spectroscopy study on a lithium sulfur pouch cell. ECS Trans. 2016, 72, 13-22. [CrossRef]

5. Xing, Y.; He, W.; Pecht, M.; Tsui, K.L. State of charge estimation of lithium-ion batteries using the open-circuit voltage at various ambient temperatures. Appl. Energy 2014, 113, 106-115. [CrossRef]

6. Chiang, Y.H.; Sean, W.Y.; Ke, J.C. Online estimation of internal resistance and open-circuit voltage of lithium-ion batteries in electric vehicles. J. Power Sour. 2011, 196, 3921-3932. [CrossRef]

7. Guo, L.; Hu, C.; Li, G. The SOC estimation of battery based on the method of improved ampere-hour and Kalman filter. In Proceedings of the 10th Conference on Industrial Electronics and Applications, Auckland, New Zealand, 15-17 June 2015; pp. 1458-1460.

8. Chen, Z.; Qiu, S.; Masrur, M.A.; Murphey, Y.L. Battery state of charge estimation based on a combined model of extended Kalman filter and neural networks. In Proceedings of the International Joint Conference on Neural Networks, San Jose, CA, USA, 31 July-5 August 2011; pp. 2156-2163.

9. Antón, J.C.A.; Nieto, P.G.; de Cos Juez, F.J.; Lasheras, F.S.; Vega, M.G.; Gutiérrez, M.R. Battery state-of-charge estimator using the SVM technique. Appl. Math. Modell. 2013, 37, 6244-6253. [CrossRef]

10. Malkhandi, S. Fuzzy logic-based learning system and estimation of state-of-charge of lead-acid battery. Eng. Appl. Artif. Intell. 2006, 19, 479-485. [CrossRef]

11. Zhang, C.; Allafi, W.; Dinh, Q.; Ascencio, P.; Marco, J. Online estimation of battery equivalent circuit model parameters and state of charge using decoupled least squares technique. Energy 2018, 142, 678-688. [CrossRef]

12. Plett, G.L. Extended Kalman filtering for battery management systems of LiPBbased HEV battery packs. Section 1. Background. J. Power Sour. 2004, 134, 252-261. [CrossRef]

13. Plett, G.L. Extended Kalman filtering for battery management systems of LiPBbased HEV battery packs. Section 2. Modeling and identification. J. Power Sour. 2004, 134, 262-276. [CrossRef]

14. Plett, G.L. Extended Kalman filtering for battery management systems of LiPBbased HEV battery packs. Section 3. State and parameter estimation. J. Power Sour. 2004, 134, 277-292. [CrossRef]

15. Plett, G.L. Sigma-point Kalman filtering for battery management systems of LiPBbased HEV battery packs: Section 2: Simultaneous state and parameter estimation. J. Power Sour. 2006, 161, 1369-1384. [CrossRef]

16. Andre, D.; Appel, C.; Soczka-Guth, T.; Sauer, T.U. Advanced mathematical methods of SOC and SOH estimation for lithium-ion batteries. J. Power Sour. 2013, 224, 20-27. [CrossRef]

17. Guo, F.; Hu, G.; Xiang, S.; Zhou, P.; Hong, R.; Xiong, N. A multi-scale parameter adaptive method for state of charge and parameter estimation of lithium-ion batteries using dual Kalman filters. Energy 2019, 178, 79-88. [CrossRef]

18. Zhang, X.; Wang, Y.; Yang, D.; Chen, Z. An on-line estimation of battery pack parameters and state-of-charge using dual filters based on pack model. Energy 2016, 115, 219-229. [CrossRef]

19. Wang, Q.; Kang, J.; Tan, Z.; Luo, M. An online method to simultaneously identify the parameters and estimate states for lithium ion batteries. Electrochim. Acta 2018, 289, 376-388. [CrossRef]

20. Chen, Z.; Yang, L.; Zhao, X.; Wang, Y.; He, Z. Online state of charge estimation of Li-ion battery based on an improved unscented Kalman filter approach. Appl. Math. Modell. 2019, 70, 532-544. [CrossRef] 
21. Wang, Q.; Wang, J.; Zhao, P.; Kang, J.; Yan, F.; Du, C. Correlation between the model accuracy and model-based SOC estimation. Electrochim. Acta 2017, 228, 146-159. [CrossRef]

22. Johnson, V.H. Battery performance models in ADVISOR. J. Power Sour. 2002, 110, 321-329. [CrossRef]

23. Simon, H. KALMAN Filtering and Neural Networks; John Wiley \& Sons: New York, NY, USA, 2001; pp. 1-10.

24. Zhang, F.; Liu, J.; Fang, J. Battery State Estimation Using Unscented Kalman Filter. In Proceedings of the IEEE International Conference on Robotics and Automation, Kobe, Japan, 12-17 May 2009.

25. Peng, S.M.; Chen, C.; Shi, H.B. State of charge estimation of battery energy storage systems based on adaptive unscented kalman filter with a noise statistics estimator. IEEE Access 2017, 5, 13202-13212. [CrossRef]

26. USCAR.Manuals: Electrical Vehicle Battery Test Procedures Manual. Available online: http://www.uscar.org/ guest/publications (accessed on 30 August 2017).

27. Xiong, R.; Yu, Q.; Wang, Y.; Lin, C. A novel method to obtain the open circuit voltage for the state of charge of lithium ion batteries in electric vehicles by using $\mathrm{H}$ infinity filter. Appl. Energy 2017, 207, 346-353. [CrossRef]

(C) 2019 by the authors. Licensee MDPI, Basel, Switzerland. This article is an open access article distributed under the terms and conditions of the Creative Commons Attribution (CC BY) license (http://creativecommons.org/licenses/by/4.0/). 